\section{RSP}

http://www.rsp.fsp.usp.br/
Revista de Saúde Pública

\title{
Adherence to physical activity in adults with chronic diseases: ELSA-Brasil
}

Ludimila Forechi', José Geraldo Mill", Rosane Härter Griep"', Itamar Santos' ${ }^{\mathrm{IV}}$, Francisco Pitangav', Maria del Carmen Bisi Molina ${ }^{\mathrm{VI}}$

1 Universidade Federal de Juiz de Fora. Departamento de Fisioterapia. Governador Valadares, MG, Brasil

" Universidade Federal do Espírito Santo. Departamento de Ciências Fisiológicas. Programa de Pós-Graduação em Saúde Coletiva. Vitória, ES, Brasil

III Fundação Oswaldo Cruz. Instituto Oswaldo Cruz. Laboratório de Educação em Ambiente e Saúde. Rio de Janeiro, RJ, Brasil

Iv Universidade de São Paulo. Faculdade de Medicina. Departamento de Clínica Médica. São Paulo, SP, Brasil

$\checkmark$ Universidade Federal da Bahia. Faculdade de Educação. Departamento de Educação Física. Salvador, BA, Brasil

Vı Universidade Federal do Espírito Santo. Departamento de Educação Integrada em Saúde. Programa de PósGraduação em Saúde Coletiva. Vitória, ES, Brasil

\section{ABSTRACT}

OBJECTIVE: The objective of this study is to investigate the adherence and the factors that influence adherence to physical activity in adults with dyslipidemia, hypertension, or diabetes.

METHODS: The analyses were based on data collected at the baseline of the 14,521 participants from the study ELSA-Brasil aged between 35 and 74 years. The level of leisure time physical activity was determined using the International Physical Activity Questionnaire. Logistic regression analyses were performed to examine the influence of the demographic data, socioeconomic conditions, perceived health status, and access to exercise facilities in the neighborhood on adherence to physical activity.

RESULTS: Men with hypertension and dyslipidemia were more active than women. The results show that $17.8 \%, 15.1 \%$, and $13.9 \%$ of the subjects who reported dyslipidemia, hypertension, and diabetes, respectively, adhere to the physical activity recommendations. The factors positively associated with adherence were higher education and income. Older individuals who reported poor perceived health, were overweight and obese, regularly smoked, and had fewer opportunities to exercise in the neighborhood presented lower adherence.

Correspondence:
Maria del Carmen Bisi Molina Av. Marechal Campos, 1468 Maruípe 29040-090 Vitória, ES, Brasil E-mail: maria.molina@ufes.br

Received: May 1, 2017

Approved: Jun 28, 2017

How to cite: Forechi L, Mill JG, Griep RH, Santos I, Pitanga F, Molina MCB. Adherence to physical activity in adults with chronic diseases: ELSA-Brasil. Rev Saude Publica. 2018;52:31.

Copyright: This is an open-access article distributed under the terms of the Creative Commons Attribution License, which permits unrestricted use, distribution, and reproduction in any medium, provided that the original author and source are credited.
CONCLUSIONS: The number of adults with dyslipidemia, hypertension, and diabetes who adhere to the physical activity recommendations is very low. Higher education and income are positively associated with adherence, while age, excess body weight, negative perceived health, regular smoking, and lack of opportunity to exercise in the neighborhood were considered barriers to physical activity.

DESCRIPTORS: Exercise. Patient Compliance. Patient Dropouts. Risk Factors. Socioeconomic. Factors Dyslipidemias, prevention \& control. Diabetes Mellitus, prevention \& control. 


\section{INTRODUCTION}

Noncommunicable chronic diseases (NCD) are the leading cause of morbidity and mortality worldwide ${ }^{1}$. Many studies have shown that regular aerobic exercises can prevent or change the natural history of several NCD, including hypertension, diabetes, and dyslipidemia. However, the number of individuals who regularly attend aerobic exercise programs is relatively low. This low adherence to regular exercise may be attributed to several factors. A better understanding of these factors is important for the healthcare system because NCD are highly prevalent in adults and because the adoption of adequate lifestyle habits, such as regular exercise, may reduce the incidence of these diseases, as well as health costs. The main fifteen global risk factors explain half the global mortality and more than a third of the global disability-adjusted life years (DALY); high levels of blood pressure, plasma glucose, and cholesterol represent the second, third, and seventh leading risk factors, respectively, in the Latin America and Caribbean countries ${ }^{2}$. Quantifying the relationships of these risk factors with overall morbidity and mortality is important because most cardiovascular outcomes associated with these factors, e.g., myocardial infarction and stroke, may be prevented with long-term adherence to physical activity (PA) programs ${ }^{3}$.

For adults, the current recommendations regarding PA are at least 150 minutes of moderateintensity aerobic PA per week, at least 75 minutes of vigorous-intensity aerobic PA per week, or an equivalent combination of the two. Aerobic activities should be performed in at least 10-minute intervals and can include leisure time physical activity (LTPA), transportation, occupational work, and housework 4 .

The benefits of regular PA for the prevention and treatment of hypertension, diabetes, and dyslipidemia are well documented. Regular PA in patients with type 2 diabetes reduces the risk of stroke, renal failure, and peripheral thrombotic events ${ }^{5}$. Aerobic exercise also improves glucose control in patients with type 2 diabetes $^{6}$. In hypertensive patients, PA has been associated with better blood pressure control and the regression or prevention of left ventricular hypertrophy and arterial stiffening. Epidemiological and experimental data also support the favorable effect of regular exercise on lipoprotein profiles and thus the prevention of atherosclerosis.

Despite these benefits, recent estimates suggest that only $31.1 \%$ of adults adhere to the actual PA recommendations ${ }^{8}$. Furthermore, although older adults have a higher prevalence of NCD and would benefit more from PA, physical inactivity is more common in this population, and adherence to PA recommendations may thus be even lower in older groups ${ }^{9}$. However, studies on the factors that influence adherence to PA recommendations are scarce, especially in the Brazilian population. Adherence is affected by a complex interaction of social, emotional, environmental, and psychological factors ${ }^{10}$, and understanding these factors is of great importance for healthcare professionals. Therefore, in this study, we sought to determine the rates of adherence to the recommended levels of LTPA and the factors that influence this adherence in participants with self-reported hypertension, diabetes, or dyslipidemia at the baseline of the Brazilian Longitudinal Study of Adult Health (ELSA-Brasil).

\section{METHODS}

\section{Study Population}

The ELSA-Brasil was designed to investigate the incidence and determinants of chronic diseases in the Brazilian population using a prospective cohort ${ }^{11}$. This cohort consisted of 15,105 civil servants (35-74 years old, active or retired) from six public teaching and research institutions in six Brazilian cities. Baseline data were collected from 2008 to 2010. The project was approved by all of the ethic committees of the institutions from which the data were collected. All participants signed the informed consent before responding to the questionnaires, which were completed during a face-to-face interview, and undergoing the 
clinical and laboratory exams. The main characteristics of the cohort at baseline have been published elsewhere ${ }^{12}$. Previous diagnoses of hypertension, diabetes, and dyslipidemia were obtained by self-report during the interview.

\section{PA Assessment}

Physical activity was determined by the LTPA domain of the International Physical Activity Questionnaire(IPAQ). This instrument has been validated in the Brazilian population and includes questions regarding the frequency, duration, and intensity of activities lasting ten or more minutes ${ }^{13}$. The IPAQ includes activities performed during leisure time and for transportation. Moderateintensity PA refers to tasks that require moderate physical effort (a small increase in breath rate compared with that at rest). Vigorous-intensity PA refers to tasks that require substantial physical and respiratory efforts. Moderate- and vigorous-intensity PA were reported in min/week.

Adherence to PA guidelines ${ }^{4}$ was defined as total PA time $\geq 150 \mathrm{~min} /$ week [(minutes of moderate-intensity PA/week) + (minutes of vigorous-intensity PA/week) $\times 2$ ]. Participants with lower values were considered non-adherent to PA recommendations.

\section{Covariates}

We tested the influence of four groups of covariates that may affect adherence to PA: demographic characteristics, socioeconomic condition, perceived health status, and access to exercise facilities in the participant's neighborhood of residence.

The demographic characteristics included age, marital status, and self-declared race. Age was categorized into decades (35-44, 45-54, 55-64, or 65-74 years old), marital status was categorized into married/living with partner, divorced/widowed, or single, and self-declared race was categorized into white or non-white.

Socioeconomic condition included years of education (less than high school, high school, or college), employment situation (active or retired), occupation (unskilled, technical/clerical, or faculty and professional staff), and monthly family income (in US\$: < 505, 505 to 1,250, or $>1,250$ ). The income of the participants was obtained in Brazilian Reais (BRL), which were converted to US\$ at a rate of BRL $2=\mathrm{US} \$ 1$.

The covariates of health status included self-perceived health (reported as very good or good, fair, or poor or very poor), smoking status (never, former, or regular), alcohol use (never, former, or regular), morbidity score (presence of diabetes, hypertension, or dyslipidemia - 1, 2, or 3 of these conditions), presence of joint disease (yes or no), and body mass index (BMI), defined as body weight divided by height squared $\left(\mathrm{kg} / \mathrm{m}^{2}\right)$. Body weight was measured with an electronic scale (Toledo, Brazil), and height was assessed with a wall-mounted stadiometer (Seca, Germany). The BMI was classified into three categories according to the WHO guidelines: underweight/normal $\left(\leq 25 \mathrm{~kg} / \mathrm{m}^{2}\right)$, overweight $\left(25-29.9 \mathrm{~kg} / \mathrm{m}^{2}\right)$, and obese $\left(\geq 30 \mathrm{~kg} / \mathrm{m}^{2}\right)^{12}$.

Three factors were investigated regarding the participant's neighborhood: opportunity to practice $\mathrm{PA}$, seeing persons practicing $\mathrm{PA}$, and facilities to practice $\mathrm{PA}^{14}$. These factors were evaluated by three consecutive statements: "My neighborhood offers many opportunities to be physically active", "I often see other people exercising in my neighborhood", and "Local sports clubs and other facilities in my neighborhood offer many opportunities to get exercise". The participant was instructed to choose the best answer among the following: 1 - strongly agree, 2 - partially agree, 3 - neither agree nor disagree, 4 - partially disagree, and 5 -strongly disagree. For this study, responses 1 and 2 were merged into "agree" and 4 and 5 were merged into "disagree".

\section{Statistical Analysis}

Categorical variables were described as absolute counts and proportions. Chi-square tests were used for the bivariate analysis of the associations between the characteristics of participants and adherence to PA recommendations. 
Logistic regression analyses were performed to estimate the crude and adjusted odds ratios (OR) and 95\% confidence intervals (95\%CI) of physical activity in men and women. The variables included in the logistic regression models were selected based on the statistical criterion of $p<0.05$ in the chi-square test. Logistic regression without adjustment (crude OR) was performed to evaluate the association of demographic characteristics, socioeconomic condition, perceived health status, and access to exercise facilities in the neighborhood with adherence to PA recommendations among subjects with self-reported dyslipidemia, hypertension, or diabetes. Considering the presence of possible confounders, six different adjusted logistic regression models were tested. In these analyses, we selected the variables that did not include zero in their $95 \% \mathrm{CI}$. All analyses were stratified by gender and performed in SPSS 18.0 (Chicago, IL, USA). The significance level was set at $\mathrm{p}<0.05$.

\section{RESULTS}

This analysis was limited to the 14,521 participants ( $96.1 \%$ of the cohort) with complete data. We excluded subjects with incomplete IPAQ $(n=223)$, those with no information regarding a previous diagnosis of hypertension $(n=4)$, diabetes $(n=10)$, or dyslipidemia $(n=31)$, and women who reported diabetes or hypertension only during pregnancy $(\mathrm{n}=316)$.

Dyslipidemia was reported by $36.0 \%$ of the participants, hypertension by $34.7 \%$, and diabetes by $8.9 \%$. A total of $36.3 \%$ of participants reported only one of these diagnoses, $16.4 \%$ reported two, and 3.5\% reported three. Most individuals (81\%) either did not practice any type of PA or practiced some PA without reaching the WHO-recommended levels. Only $19.0 \%$ of the cohort adhered to the PA recommendations, and there were minor differences among those who reported having high cholesterol (17.8\%), hypertension (15.1\%), or diabetes (13.9\%). Men were more likely to be active than women only among individuals who self-reported dyslipidemia or hypertension (Figure).

\section{Characteristics of the Sample According to Adherence to PA}

The effects of the demographic characteristics, socioeconomic conditions, health status, and access on adherence to PA are shown in Table 1. Demographic variables (age, marital status, and race [white versus non-white]) showed little or no influence in general, except among younger men with dyslipidemia or hypertension, who were more likely to be adherent than their older counterparts. Two socioeconomic variables (education level and income) were closely related to adherence to PA, while the other two (occupation level and employment) were unrelated to adherence. Higher education and higher income were associated with

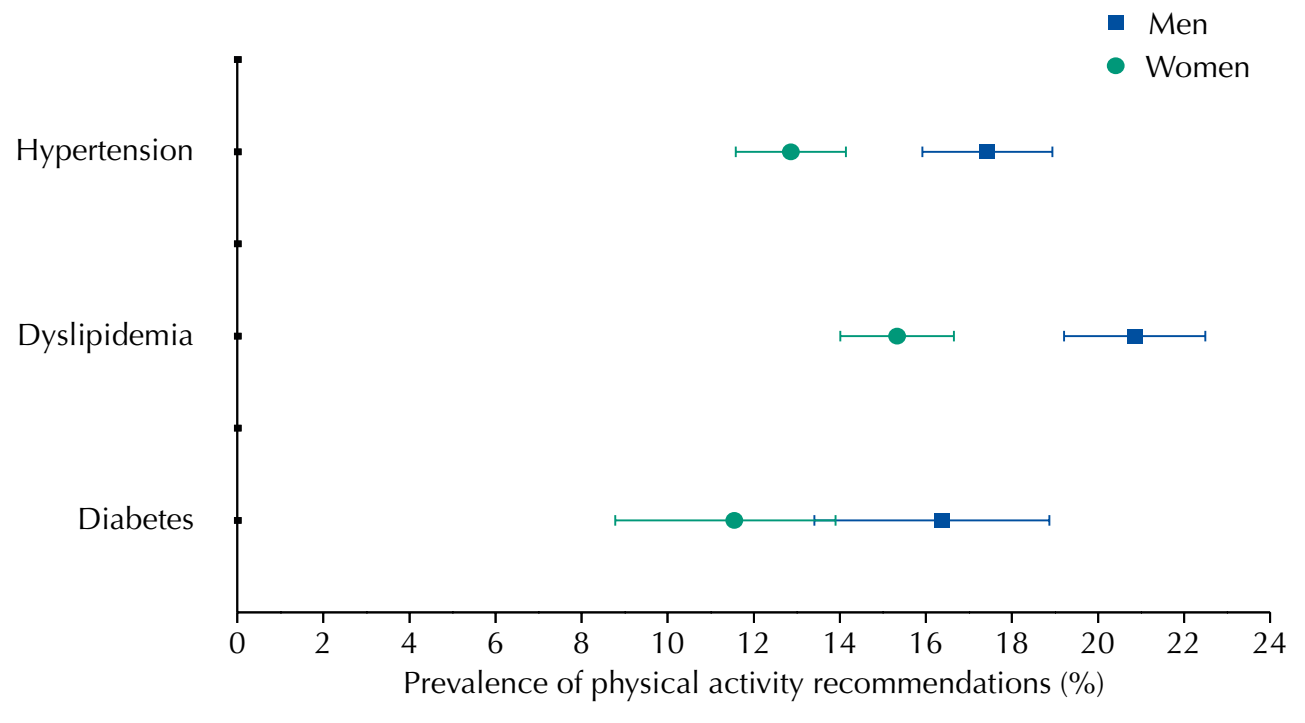

Figure. Prevalence of adherence to physical activity recommendations by gender. 
Table 1. Demographic characteristics, socioeconomic condition, perceived health status, and access to exercise among subjects with dyslipidemia, hypertension, and diabetes meeting the physical activity (PA) recommendations in the ELSA-Brasil cohort.

\begin{tabular}{|c|c|c|c|c|c|c|c|c|c|c|c|c|}
\hline \multirow{3}{*}{ Variable } & \multicolumn{4}{|c|}{ Dyslipidemia $(n=5,232)$} & \multicolumn{4}{|c|}{ Hypertension $(n=5,040)$} & \multicolumn{4}{|c|}{ Diabetes $(n=1,291)$} \\
\hline & \multicolumn{2}{|c|}{$\begin{array}{c}\text { Men } \\
(n=2,369) \\
\end{array}$} & \multicolumn{2}{|c|}{$\begin{array}{c}\text { Women } \\
(n=2,863) \\
\end{array}$} & \multicolumn{2}{|c|}{$\begin{array}{c}\text { Men } \\
(n=2,427)\end{array}$} & \multicolumn{2}{|c|}{$\begin{array}{c}\text { Women } \\
(n=2,613) \\
\end{array}$} & $\begin{array}{r}\text { Me } \\
(n=7\end{array}$ & $\begin{array}{l}\text { en } \\
700) \\
\end{array}$ & $\begin{array}{c}\text { Wor } \\
(\mathrm{n}=\end{array}$ & $\begin{array}{l}\text { nen } \\
591)\end{array}$ \\
\hline & n (\%) & $p$ & n (\%) & $p$ & n (\%) & $p$ & n (\%) & $\mathbf{p}$ & n (\%) & $p$ & n (\%) & $p$ \\
\hline & & & & Demogr & raphic chara & acteristics & & & & & & \\
\hline Age group (years) & & $<0.001$ & & 0.182 & & $<0.001$ & & 0.595 & & 0.474 & & 0.662 \\
\hline $35-44$ & $98(27.3)$ & & $46(13.9)$ & & $61(23.6)$ & & $25(10.2)$ & & $8(16.3)$ & & $2(6.9)$ & \\
\hline $45-54$ & $200(21.6)$ & & $154(15.5)$ & & $156(18.4)$ & & $117(13.2)$ & & $36(16.8)$ & & $22(12.9)$ & \\
\hline $55-64$ & $128(17.0)$ & & $184(16.7)$ & & $112(13.0)$ & & $137(13.3)$ & & $40(13.8)$ & & $25(10.1)$ & \\
\hline $65-74$ & 68 (20.5) & & $55(12.5)$ & & $94(20.5)$ & & 57 (12.6) & & 29 (19.6) & & $18(12.5)$ & \\
\hline Marital status & & 0.521 & & 0.812 & & 0.409 & & 0.285 & & 0.605 & & 0.075 \\
\hline Married/Union & $408(20.8)$ & & $211(15.2)$ & & $361(17.8)$ & & $172(14.0)$ & & $94(16.4)$ & & $33(12.0)$ & \\
\hline Divorced & $66(22.6)$ & & $155(15.0)$ & & $42(14.6)$ & & $119(11.9)$ & & $13(13.4)$ & & $19(8.3)$ & \\
\hline Single & $20(17.5)$ & & $73(16.3)$ & & $20(18.3)$ & & $45(11.8)$ & & $6(20.7)$ & & $15(17.2)$ & \\
\hline Race & & 0.010 & & 0.277 & & 0.435 & & 0.947 & & 0.496 & & 0.357 \\
\hline White & $271(23.0)$ & & $222(14.7)$ & & $214(18.0)$ & & $180(12.8)$ & & $59(17.3)$ & & $33(10.2)$ & \\
\hline Non-white & $217(18.7)$ & & $212(16.2)$ & & $(16.8)$ & & $150(12.7)$ & & $54(15.3)$ & & $33(12.6)$ & \\
\hline & & & & Socioe & economic co & ondition & & & & & & \\
\hline Years of education & & $<0.001$ & & $<0.001$ & & $<0.001$ & & $<0.001$ & & $<0.001$ & & 0.010 \\
\hline Less than high school & $19(9.6)$ & & $5(3.3)$ & & $22(8.4)$ & & $8(4.2)$ & & $5(4.4)$ & & $1(1.7)$ & \\
\hline High school & $24(12.2)$ & & $13(7.1)$ & & $22(9.3)$ & & $22(10.2)$ & & $10(13.3)$ & & $4(6.0)$ & \\
\hline College & $451(22.8)$ & & $421(16.7)$ & & $379(19.7)$ & & $306(13.9)$ & & $98(19.2)$ & & $62(13.3)$ & \\
\hline Employment situation & & 0.439 & & 0.420 & & 0.334 & & 0.109 & & 0.060 & & 0.427 \\
\hline Active & $397(21.2)$ & & $284(14.9)$ & & $306(17.0)$ & & $205(12.1)$ & & $69(14.4)$ & & $33(10.4)$ & \\
\hline Retired & 97 (19.6) & & $155(16.1)$ & & $117(18.7)$ & & $131(14.3)$ & & $44(20.0)$ & & $34(12.5)$ & \\
\hline Occupation & & 0.149 & & 0.904 & & 0.358 & & 0.193 & & 0.350 & & 0.618 \\
\hline Unskilled & $127(20.0)$ & & $132(15.4)$ & & $111(17.0)$ & & $100(13.8)$ & & $34(19.0)$ & & $17(10.9)$ & \\
\hline Technical/Clerical & $171(19.4)$ & & $147(14.9)$ & & $151(16.4)$ & & $107(11.3)$ & & 38 (13.9) & & $28(13.0)$ & \\
\hline Faculty/Professional staff & $196(23.0)$ & & $160(15.7)$ & & $161(18.9)$ & & $129(13.7)$ & & $41(16.5)$ & & $22(10.0)$ & \\
\hline Per capita income (USD) & & $<0.001$ & & $<0.001$ & & $<0.001$ & & $<0.001$ & & $<0.001$ & & $<0.001$ \\
\hline$<505.00$ & $123(14.5)$ & & $76(8.1)$ & & $134(13.6)$ & & $90(8.6)$ & & $30(9.6)$ & & $12(5.1)$ & \\
\hline $505.00-1,250.00$ & $200(21.5)$ & & $178(15.4)$ & & $159(17.8)$ & & $120(12.0)$ & & $39(17.0)$ & & $32(13.4)$ & \\
\hline$>1,250.00$ & $170(29.1)$ & & $182(24.5)$ & & $129(23.7)$ & & $126(22.9)$ & & $43(28.9)$ & & $23(20.0)$ & \\
\hline & & & Healtl & h statu & and health-re & elated beh & haviors & & & & & \\
\hline Self-perceived health & & $<0.001$ & & $<0.001$ & & $<0.001$ & & $<0.001$ & & 0.002 & & 0.002 \\
\hline Very good/Good & $417(23.7)$ & & $371(17.8)$ & & $344(21.0)$ & & $267(15.1)$ & & $80(20.3)$ & & $45(16.0)$ & \\
\hline Fair & $70(12.8)$ & & $63(9.4)$ & & $69(9.7)$ & & $68(9.3)$ & & $26(10.0)$ & & $20(7.8)$ & \\
\hline Poor/Very poor & $6(9.7)$ & & $5(4.5)$ & & $9(12.5)$ & & $1(0.9)$ & & $6(14.0)$ & & $2(3.8)$ & \\
\hline Smoking status & & $<0.001$ & & 0.046 & & 0.010 & & 0.060 & & 0.375 & & 0.305 \\
\hline Never & $259(24.2)$ & & $273(15.8)$ & & $204(20.0)$ & & $211(13.2)$ & & $48(18.7)$ & & $46(13.0)$ & \\
\hline Former & $188(19.1)$ & & $129(16.2)$ & & $175(16.3)$ & & $100(13.9)$ & & $51(15.0)$ & & $15(8.6)$ & \\
\hline Regular & $47(14.8)$ & & $37(10.8)$ & & $44(13.4)$ & & $25(8.6)$ & & $14(13.9)$ & & $6(9.7)$ & \\
\hline Alcohol use & & 0.005 & & $<0.001$ & & $<0.001$ & & $<0.001$ & & 0.005 & & 0.230 \\
\hline Never & $12(13.0)$ & & $55(11.4)$ & & $11(10.6)$ & & $52(10.4)$ & & $7(17.5)$ & & $21(14.3)$ & \\
\hline Former & $68(16.3)$ & & $81(12.5)$ & & $68(13.0)$ & & $61(9.3)$ & & $20(9.3)$ & & $15(8.3)$ & \\
\hline Regular & $413(22.2)$ & & $303(17.6)$ & & $343(19.1)$ & & $223(15.4)$ & & $85(19.2)$ & & $31(11.8)$ & \\
\hline Morbidity score & & $<0.001$ & & $<0.001$ & & 0.125 & & 0.017 & & 0.245 & & 0.053 \\
\hline 1 & $286(24.3)$ & & $265(17.2)$ & & $193(17.4)$ & & $152(12.5)$ & & 25 (16.9) & & $11(12.5)$ & \\
\hline 2 & $174(18.6)$ & & $154(14.4)$ & & $196(18.5)$ & & $164(14.3)$ & & $54(18.4)$ & & $36(14.6)$ & \\
\hline 3 & $34(13.2)$ & & $20(7.8)$ & & $34(13.2)$ & & $20(7.8)$ & & $34(13.2)$ & & $20(7.8)$ & \\
\hline Joint diseases & & 0.117 & & 0.003 & & 0.948 & & 0.008 & & 0.821 & & 0.239 \\
\hline No & $396(20.3)$ & & $312(16.8)$ & & $347(17.5)$ & & $236(14.2)$ & & $93(16.3)$ & & 45 (12.6) & \\
\hline Yes & $97(23.7)$ & & $127(12.7)$ & & $76(17.4)$ & & $100(10.6)$ & & $20(15.5)$ & & $22(9.5)$ & \\
\hline Body mass index & & 0.006 & & $<0.001$ & & 0.036 & & $<0.001$ & & 0.446 & & 0.401 \\
\hline Underweight/Normal & 165 (24.9) & & $195(20.9)$ & & $105(19.7)$ & & $116(19.8)$ & & 25 (17.9) & & $15(15.2)$ & \\
\hline Overweight & $224(20.0)$ & & $165(14.7)$ & & $204(18.3)$ & & $126(12.4)$ & & $58(17.1)$ & & $23(11.1)$ & \\
\hline Obese & $105(17.9)$ & & $79(9.8)$ & & $114(14.7)$ & & $94(9.3)$ & & $30(13.6)$ & & $29(10.2)$ & \\
\hline & & & & ccess to e & xercise in $\mathrm{n}$ & eighborhc & ood & & & & & \\
\hline Opportunity to practice PA & & 0.018 & & $<0.001$ & & $<0.001$ & & $<0.001$ & & 0.013 & & 0.120 \\
\hline Agree & $388(22.2)$ & & $365(17.4)$ & & $340(19.6)$ & & $268(14.7)$ & & $92(18.8)$ & & $51(13.3)$ & \\
\hline Disagree & $97(17.4)$ & & $69(10.0)$ & & $74(12.2)$ & & $61(8.6)$ & & $19(10.6)$ & & $14(7.7)$ & \\
\hline Neither agree nor disagree & $9(13.8)$ & & $5(7.6)$ & & $9(11.1)$ & & $7(8.9)$ & & $2(6.5)$ & & $2(7.7)$ & \\
\hline See persons practicing PA & & 0.725 & & 0.001 & & 0.603 & & 0.006 & & 0.143 & & 0.017 \\
\hline Agree & $403(21.0)$ & & $360(16.5)$ & & $345(17.8)$ & & $278(14.0)$ & & $99(17.4)$ & & $53(11.9)$ & \\
\hline Disagree & $72(19.5)$ & & $65(10.6)$ & & $63(15.7)$ & & $51(9.1)$ & & $11(9.9)$ & & $9(6.9)$ & \\
\hline Neither agree nor disagree & $18(22.8)$ & & $14(19.2)$ & & $14(16.9)$ & & $7(10.0)$ & & $3(15.0)$ & & $5(29.4)$ & \\
\hline Conditions to practice PA & & 0.187 & & $<0.001$ & & 0.029 & & 0.004 & & 0.449 & & 0.290 \\
\hline Agree & $366(21.9)$ & & $325(17.3)$ & & $314(18.8)$ & & $244(14.4)$ & & $83(17.3)$ & & $46(13.0)$ & \\
\hline Disagree & 104 (18.6) & & $90(11.0)$ & & $92(14.5)$ & & $74(9.6)$ & & $25(14.2)$ & & $18(9.1)$ & \\
\hline Neither agree nor disagree & $24(17.8)$ & & $24(14.7)$ & & $17(14.0)$ & & $18(12.3)$ & & 5 (11.6) & & $3(7.7)$ & \\
\hline
\end{tabular}


higher adherence in both men and women. Self-perceived health showed a direct relationship with adherence for all three access conditions in both genders; that is, those who reported having a lower health status were less likely to adhere to PA recommendations. Participants who complained of joint diseases, as well as those with higher body fat, were also less likely to be adherent. An interesting finding was observed in relation to smoking and alcohol intake. Specifically, adherence to PA was less likely among regular smokers, except for those who reported diabetes, whereas regular use of alcohol was associated with adherence to PA in all groups, except for women who reported diabetes. Finally, subjects with more than one risk factor were less likely to adhere to PA recommendations, regardless of gender.

Our results clearly showed a correlation between the availability of facilities in the neighborhood and adherence to PA. Adherence was higher in those who agreed that neighborhood facilities for sports were important and in women who frequently observed persons exercising (Table 1).

\section{Factors Related to Adherence to PA Recommendations}

Tables 2 and 3 show the OR and 95\%CI for the associations of demographic characteristics, socioeconomic condition, perceived health status, and health perceptions with adherence to $\mathrm{PA}$ recommendations by gender.

Both men and women with high cholesterol were less likely to meet the recommendations if they were overweight or obese, were current smokers, had a fair or poor/very poor self-perceived health status, and did not have the opportunity to practice PA in their neighborhood. In contrast, regular use of alcohol, high education level, and high per capita income showed positive associations with adherence to PA. Among men with dyslipidemia, those who were older than 44 years and who were non-white also showed decreased adherence to PA. Additionally, women who had joint diseases, did not have facilities to practice PA, and did not see persons practicing PA were less likely to be adherent. After adjusting the models for all positive and negative factors that interfered with adherence to PA in men and women with dyslipidemia, we observed a significant positive association in both genders for only high income and negative associations for fair self-perceived health, regular smoking status, and overweight or obesity. The positive association between adherence to PA and university education was confirmed only for women. Non-white males with dyslipidemia were 0.77 (95\%CI 0.63-0.95) times less likely to be adherent than their white counterparts. As expected, age was inversely related to adherence to PA in men.

In men with hypertension, an age of 55-64 years ( $\mathrm{OR}=0.49$, 95\%CI 0.34-0.69), fair self-perceived health $(\mathrm{OR}=0.40,95 \% \mathrm{CI} 0.31-0.53)$, regular smoking status $(\mathrm{OR}=0.62$, 95\%CI $0.44-0.88$ ), obesity ( $\mathrm{OR}=0.70,95 \% \mathrm{CI} 0.52-0.94)$, lack of opportunity to practice $\mathrm{PA}(\mathrm{OR}=0.57,95 \% \mathrm{CI} 0.44-0.75)$, and lack of conditions to practice $\mathrm{PA}(\mathrm{OR}=0.73,95 \% \mathrm{CI}$ $0.57-0.94)$ were factors that contributed to poor adherence to PA. In contrast, a university education $(\mathrm{OR}=2.67,95 \% \mathrm{CI} 1.70-4.19)$, the highest category of income $(\mathrm{OR}=1.96,95 \% \mathrm{CI}$ $1.50-2.57)$, and regular alcohol use $(\mathrm{OR}=1.99,95 \% \mathrm{CI} 1.05-3.76)$ were factors that positively contributed to adherence to PA in men. After adjustment for all of those factors, similar results were found except for years of education, alcohol use, and conditions to practice PA in the neighborhood. In the crude analyses, years of education, income, alcohol use, BMI, and opportunity and conditions to engage in PA led to the same behavior in women with hypertension as in men with hypertension. However, individuals with poor or very poor self-perceived health had lower adherence. Other factors that contributed to poor adherence to PA in hypertensive women included the presence of three risk factors $(\mathrm{OR}=0.59,95 \% \mathrm{CI}$ $0.36-0.96)$, the presence of joint disease ( $\mathrm{OR}=0.71,95 \% \mathrm{CI} 0.56-0.92)$, and the lack of seeing other persons exercising $(\mathrm{OR}=0.61,95 \% \mathrm{CI} 0.44-0.83)$. After adjusting for all of the factors previously mentioned, we observed a significant negative association between adherence to PA and only poor self-perceived health. In the opposite direction, we observed significant positive associations of adherence to PA in hypertensive women with high educational level and income. 
Table 2. Odds ratios and 95\% confidence intervals for demographic and socioeconomic factors, health status, lifestyle, and health-related behaviors associated with adherence to physical activity recommendations in men.

\begin{tabular}{|c|c|c|c|c|c|c|c|c|c|c|c|c|}
\hline \multirow{3}{*}{ Variable } & \multicolumn{12}{|c|}{ Crude and adjusted odds ratios in men } \\
\hline & \multicolumn{4}{|c|}{$\begin{array}{c}\text { Self-reported Dyslipidemia } \\
(\mathrm{n}=2,369)\end{array}$} & \multicolumn{4}{|c|}{$\begin{array}{c}\text { Self-reported Hypertension } \\
(\mathrm{n}=2,427) \\
\end{array}$} & \multicolumn{4}{|c|}{$\begin{array}{l}\text { Self-reported Diabetes } \\
\qquad(\mathrm{n}=700)\end{array}$} \\
\hline & C OR & $95 \% \mathrm{Cl}$ & A OR & $95 \% \mathrm{Cl}$ & COR & $95 \% \mathrm{Cl}$ & A OR & $95 \% \mathrm{Cl}$ & C OR & $95 \% \mathrm{Cl}$ & A OR & $95 \% \mathrm{Cl}$ \\
\hline
\end{tabular}

\begin{tabular}{ccccccccc}
\hline $\begin{array}{c}\text { Age (years) } \\
35-44\end{array}$ & 1 & & 1 & & 1 & & & \\
$45-54$ & 0.73 & $0.55-0.97$ & 0.88 & $0.65-1.19$ & 0.73 & $0.52-1.02$ & 0.74 & $0.52-1.05$ \\
$55-64$ & 0.55 & $0.40-0.74$ & 0.61 & $0.44-0.85$ & 0.49 & $0.34-0.69$ & 0.45 & $0.31-0.66$ \\
$65-74$ & 0.69 & $0.48-0.98$ & 0.65 & $0.44-0.98$ & 0.84 & $0.58-1.21$ & 0.68 & $0.45-1.01$
\end{tabular}

Race

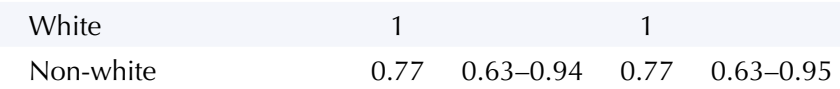

\begin{tabular}{|c|c|c|c|c|c|c|c|c|c|c|c|c|}
\hline \multicolumn{13}{|c|}{ Socioeconomic conditions } \\
\hline \multicolumn{13}{|l|}{ Years of education } \\
\hline Less than high school & 1 & & 1 & & 1 & & 1 & & 1 & & 1 & \\
\hline High school & 1.31 & $0.69-2.49$ & 1.15 & $0.60-2.20$ & 1.12 & $0.60-2.07$ & 0.94 & $0.50-1.77$ & 3.35 & $1.10-10.24$ & 2.86 & $0.92-8.87$ \\
\hline College & 2.79 & $1.72-4.53$ & 1.45 & $0.86-2.43$ & 2.67 & $1.70-4.19$ & 1.56 & $0.96-2.53$ & 5.17 & 2.05-13.02 & 2.66 & $1.00-7.02$ \\
\hline \multicolumn{13}{|l|}{ Per capita income (USD) } \\
\hline$<505.00$ & 1 & & 1 & & 1 & & 1 & & 1 & & 1 & \\
\hline $505.00-1,250.00$ & 1.61 & $1.26-2.06$ & 1.32 & $1.01-1.72$ & 1.37 & $1.07-1.76$ & 1.04 & $0.79-1.37$ & 1.93 & $1.16-3.22$ & 1.50 & $0.87-2.58$ \\
\hline$>1,250.00$ & 2.41 & $1.85-3.13$ & 1.92 & $1.43-2.60$ & 1.96 & $1.50-2.57$ & 1.39 & $1.01-1.91$ & 3.84 & $2.29-6.44$ & 2.57 & $1.45-4.55$ \\
\hline \multicolumn{13}{|c|}{ Health status and health-related behaviors } \\
\hline \multicolumn{13}{|l|}{ Self-perceived health } \\
\hline Very good/Good & 1 & & 1 & & 1 & & 1 & & 1 & & 1 & \\
\hline Fair & 0.47 & $0.36-0.62$ & 0.60 & $0.44-0.80$ & 0.40 & $0.31-0.53$ & 0.50 & $0.37-0.66$ & 0.44 & $0.27-0.70$ & 0.55 & $0.33-0.91$ \\
\hline Poor/Very poor & 0.34 & $0.15-0.80$ & 0.50 & $0.21-1.20$ & 0.54 & $0.26-1.09$ & 0.71 & $0.34-1.45$ & 0.64 & $0.26-1.56$ & 0.87 & $0.34-2.18$ \\
\hline \multicolumn{13}{|l|}{ Smoking status } \\
\hline Never & 1 & & 1 & & 1 & & 1 & & & & & \\
\hline Former & 0.74 & $0.60-0.91$ & 0.87 & $0.69-1.10$ & 0.78 & $0.62-0.97$ & 0.89 & $0.70-1.12$ & - & - & - & - \\
\hline Regular & 0.54 & $0.39-0.76$ & 0.60 & $0.42-0.85$ & 0.62 & $0.44-0.88$ & 0.69 & $0.48-0.99$ & & & & \\
\hline \multicolumn{13}{|l|}{ Alcohol use } \\
\hline Never & 1 & & 1 & & 1 & & 1 & & 1 & & & \\
\hline Former & 1.30 & $0.67-2.51$ & 1.27 & $0.64-2.50$ & 1.27 & $0.64-2.49$ & 1.34 & $0.67-2.68$ & 0.48 & $0.19-1.23$ & - & - \\
\hline Regular & 1.90 & $1.03-3.53$ & 1.64 & $0.87-3.10$ & 1.99 & $1.05-3.76$ & 1.73 & $0.90-3.34$ & 1.12 & $0.48-2.62$ & & \\
\hline \multicolumn{13}{|l|}{ Morbidity score } \\
\hline 1 & 1 & & 1 & & & & & & & & & \\
\hline 2 & 0.71 & $0.58-0.88$ & 0.91 & $0.72-1.14$ & - & - & - & - & - & - & - & - \\
\hline 3 & 0.47 & $0.32-0.69$ & 0.84 & $0.54-1.29$ & & & & & & & & \\
\hline \multicolumn{13}{|l|}{ Body mass index } \\
\hline Underweight/Normal & 1 & & 1 & & 1 & & 1 & & & & & \\
\hline Overweight & 0.75 & $0.60-0.95$ & 0.75 & $0.59-0.95$ & 0.91 & $0.70-1.19$ & 0.87 & $0.66-1.14$ & - & - & - & - \\
\hline Obese & 0.66 & $0.50-0.87$ & 0.71 & $0.53-0.96$ & 0.70 & $0.52-0.94$ & 0.68 & $0.50-0.92$ & & & & \\
\hline
\end{tabular}
Access to exercise in neighborhood

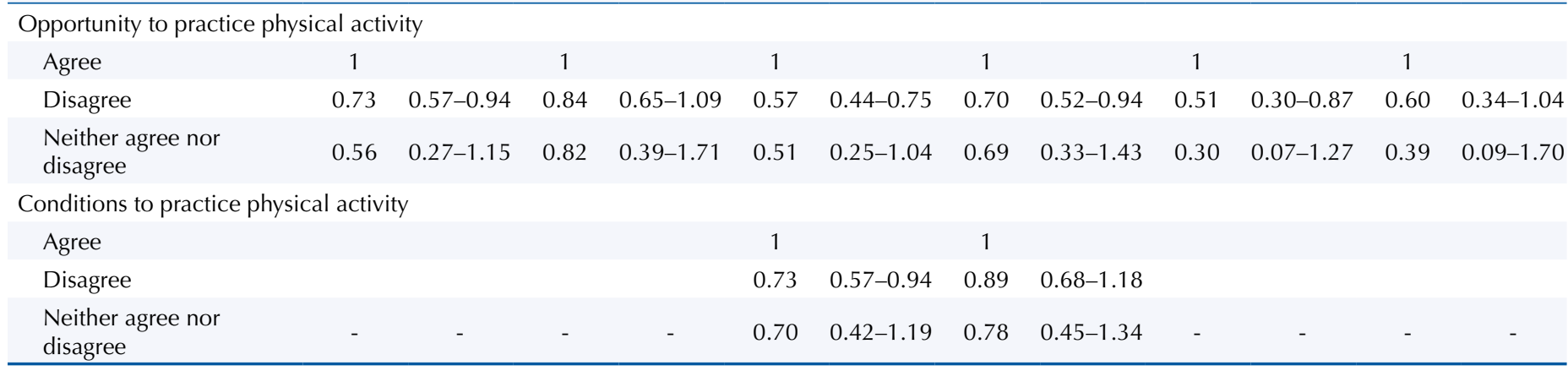

C OR: Crude OR indicates univariate logistic regression without adjustment for variables; A OR: Adjusted OR was obtained from multivariate analyses adjusted for all significant factors in the crude analysis 
Table 3. Odds ratios and 95\% confidence intervals for demographic and socioeconomic factors, health status, lifestyle, and health-related behaviors associated with adherence to physical activity recommendations in women.

\begin{tabular}{|c|c|c|c|c|c|c|c|c|c|c|c|c|}
\hline \multirow{3}{*}{ Variable } & \multicolumn{12}{|c|}{ Crude and adjusted odds ratios in women } \\
\hline & \multicolumn{4}{|c|}{$\begin{array}{l}\text { Self-reported dyslipidemia } \\
\qquad(\mathrm{n}=2,863)\end{array}$} & \multicolumn{4}{|c|}{$\begin{array}{l}\text { Self-reported hypertension } \\
\qquad(n=2,613)\end{array}$} & \multicolumn{4}{|c|}{$\begin{array}{l}\text { Self-reported diabetes } \\
\qquad(n=591)\end{array}$} \\
\hline & C OR & $95 \% \mathrm{Cl}$ & A OR & $95 \% \mathrm{Cl}$ & C OR & $95 \% \mathrm{Cl}$ & A OR & $95 \% \mathrm{Cl}$ & C OR & $95 \% \mathrm{Cl}$ & A OR & $95 \% \mathrm{Cl}$ \\
\hline
\end{tabular}

Years of education

Less than high school

High school

College

1

$2.26 \quad 0.79-6.50$

$5.92 \quad 2.41-14.51 \quad 3.02$

2.01

Per capita income (USD)

$<505.00$

$505.00-1,250.00$

1

$2.06 \quad 1.55-2.74 \quad 1.53$

1

$>1,250.00$

$3.69 \quad 2.77-4.92 \quad 2.49$

Health status and health-related behaviors

Self-perceived health

Very good/Good

Fair

Poor/Very poor

1

0.48

0.22

$0.09-0.54$

1

$0.54-0.99$

1

moking status

\begin{tabular}{lcccc} 
Never & 1 & \multicolumn{3}{c}{1} \\
Former & 1.02 & $0.81-1.29$ & 1.05 & $0.82-1.33$ \\
Regular & 0.64 & $0.45-0.93$ & 0.65 & $0.45-0.95$
\end{tabular}

Alcohol use

\begin{tabular}{lcccccccc} 
Never & 1 & & & \multicolumn{3}{c}{1} \\
Former & 1.11 & $0.77-1.60$ & 0.97 & $0.66-1.42$ & 0.88 & $0.59-1.29$ & 0.81 & $0.54-1.21$ \\
Regular & 1.65 & $1.21-2.24$ & 1.15 & $0.83-1.59$ & 1.56 & $1.14-2.16$ & 1.18 & $0.84-1.66$
\end{tabular}

Morbidity score

$\begin{array}{ccccccccc}1 & 1 & & 1 & & 1 & & \\ 2 & 0.81 & 0.65-1.00 & 1.15 & 0.91-1.45 & 1.17 & 0.92-1.48 & 1.29 & 1.01-1.65 \\ 3 & 0.41 & 0.25-0.65 & 0.83 & 0.50-1.39 & 0.59 & 0.36-0.96 & 0.96 & 0.57-1.60\end{array}$

Articular disease

\begin{tabular}{|c|c|c|c|c|c|}
\hline No & 1 & \multicolumn{3}{|c|}{1} & 1 \\
\hline Yes & 0.72 & $0.57-0.90$ & 0.93 & $0.73-1.17$ & 0.7 \\
\hline
\end{tabular}

Body mass index

\begin{tabular}{lcccccccc} 
Underweight/Normal & 1 & & 1 & & 1 & \multicolumn{3}{c}{1} \\
Overweight & 0.65 & $0.52-0.82$ & 0.71 & $0.56-0.90$ & 0.58 & $0.44-0.76$ & 0.58 & $0.44-0.78$ \\
Obese & 0.41 & $0.31-0.55$ & 0.53 & $0.39-0.71$ & 0.41 & $0.31-0.56$ & 0.50 & $0.36-0.67$
\end{tabular}

\begin{tabular}{|c|c|c|c|c|c|c|c|c|c|c|c|c|}
\hline \multicolumn{13}{|c|}{ Access to exercise in neighborhood } \\
\hline \multicolumn{13}{|c|}{ Opportunity to practice physical activity } \\
\hline Agree & 1 & & 1 & & 1 & & 1 & & & & & \\
\hline Disagree & 0.53 & $0.40-0.69$ & 0.88 & $0.63-1.19$ & 0.54 & $0.41-0.73$ & 0.83 & $0.59-1.16$ & - & - & - & - \\
\hline $\begin{array}{l}\text { Neither agree nor } \\
\text { disagree }\end{array}$ & 0.39 & $0.16-0.98$ & 0.57 & $0.22-1.48$ & 0.56 & $0.26-1.24$ & 0.78 & $0.34-1.77$ & & & & \\
\hline \multicolumn{13}{|c|}{ See persons practicing physical activity } \\
\hline Agree & 1 & & 1 & & 1 & & 1 & & 1 & & 1 & \\
\hline Disagree & 0.60 & $0.45-0.79$ & 0.81 & $0.58-1.12$ & 0.61 & $0.44-0.83$ & 0.80 & $0.56-1.15$ & 0.55 & $0.26-1.14$ & 0.57 & $0.27-1.21$ \\
\hline $\begin{array}{l}\text { Neither agree nor } \\
\text { disagree }\end{array}$ & 1.20 & $0.66-2.17$ & 1.42 & $0.74-2.71$ & 0.68 & $0.31-1.50$ & 0.65 & $0.29-1.49$ & 3.07 & $1.04-9.07$ & 2.87 & $0.91-8.99$ \\
\hline \multicolumn{13}{|c|}{ Conditions to practice physical activity } \\
\hline Agree & 1 & & 1 & & 1 & & 1 & & & & & \\
\hline Disagree & 0.59 & $0.46-0.76$ & 0.74 & $0.55-0.99$ & 0.63 & $0.48-0.83$ & 0.81 & $0.59-1.10$ & - & - & - & - \\
\hline $\begin{array}{l}\text { Neither agree nor } \\
\text { disagree }\end{array}$ & 0.83 & $0.53-1.30$ & 1.00 & $0.62-1.63$ & 0.84 & $0.50-1.39$ & 1.05 & $0.61-1.80$ & & & & \\
\hline
\end{tabular}

C OR: Crude OR indicates univariate logistic regression without adjustment for variables; A OR: Adjusted OR was obtained from multivariate analyses adjusted for all significant factors in the crude analysis 
Among those with diabetes, having a university education and income above USD 505.00 increased the odds of adherence to the PA recommended levels, and having a fair self-perceived health status lowered these odds. In the stratified analysis by gender, men who had the no opportunity to practice PA were 49 times less likely to be adherent to PA than those with the opportunity; additionally, diabetic women who neither agreed nor disagreed to seeing persons practicing PA were three times more likely to adhere to PA than diabetic women who agreed. In the multivariable models, only high income had a significant association with adherence to PA in women. Overall, high income and high education level contributed positively to adherence to PA, while a fair self-perceived health status contributed negatively.

\section{DISCUSSION}

Despite the importance of regular PA for the prevention and treatment of many chronic diseases, few studies have investigated the factors that affect adherence to PA in subjects with these diseases ${ }^{15}$. Notably, these factors may vary across populations, and such differences should be taken into account by health providers and professionals when planning and supervising exercise activities. The results of this study showed that there is low (less than $20 \%$ ) adherence to PA recommendations in adults with a previous diagnosis of dyslipidemia, hypertension, or diabetes. Similar results have been found by Fang et al..$^{16}(43.1 \%$ versus $51.7 \%)$ and Churilla et al. ${ }^{7}$ (59.1\% versus $68.3 \%$ ), who have reported that dyslipidemic adults were less likely to regularly engage in PA than those without dyslipidemia. Hays and Clark ${ }^{17}$ have also observed a low prevalence of PA in adults with type 2 diabetes.

The WHO has promoted regular and long-term PA as an intervention that can reduce the global rates of morbidity and mortality from cardiovascular diseases. Accordingly, the Global Action Plan for the Prevention and Control of NCD 2013-2020 aims to achieve a 10\% reduction in the rates of physical inactivity ${ }^{18}$. It is important to note that the ELSA-Brasil cohort study is not representative of the Brazilian population, as the participants had a higher education level and income than the general population. Despite these characteristics, our data showed that only a small proportion of the subjects of the study regularly participated in PA programs. A better understanding of the variables explaining this low adherence may help health providers in the development of strategies to encourage specific populations to improve their adherence to exercises, especially considering the large and growing body of evidence demonstrating the numerous health benefits of regular PA. Physical activity is associated with a reduced risk of all-cause mortality in hypertensive adults and a reduced incidence of cardiovascular events, microvascular complications, and all-cause mortality in diabetic patients ${ }^{19,20}$. Madden et al. ${ }^{21}$ have shown that relatively short aerobic exercise interventions in older adults reduced the arterial stiffening associated with aging, diabetes, and hypercholesterolemia and they should thus be included as first-line treatment for these conditions. Additionally, PA is a key determinant of energy expenditure and therefore plays a fundamental role in weight control ${ }^{22-24}$. Considering the high prevalence of overweight and obesity in Brazil and the world ${ }^{25}$, it is important to increase the number of individuals who regularly attend PA programs to reduce the increasing incidence of diabetes and other weight-related chronic diseases.

A number of different factors can interact to create barriers to adequate exercise. In our study, adherence to PA recommendations was lower for those aged 35 to 64 years, although it was greater in men aged 65-74 years. However, a recent study with men and women aged 70-93 years from 25 towns in the United Kingdom has found that few participants reached the current PA guidelines. Those who adhered to the guidelines were younger, had fewer chronic health conditions, and had less severe mobility limitations ${ }^{26}$. We also observed that decreased mobility from joint diseases was a limiting factor for adherence to PA in dyslipidemic women.

The male gender, a higher socioeconomic condition, and a higher education level have been reported to increase LTPA in Brazilian individuals ${ }^{27}$. Our data confirmed this finding and demonstrated that women were less active than men regardless of health condition. Another 
study showed that men were more likely to be engaged in PA at guideline-recommended levels8. Regarding socioeconomic condition, our results showed that the adherence to PA was higher in individuals with university education and in those in the highest income group. In the fully adjusted model, education and high income were the only factors that favored adherence to PA in dyslipidemic, hypertensive, and diabetic individuals. In support of our results, Dontje et al. ${ }^{28}$ have shown in a longitudinal study of middle-aged women with chronic diseases that the odds of being inactive were lower among those with a higher educational level. The gender differences found in our study are worth noting because they may suggest the need to adopt different strategies to improve adherence to PA in men and women; this type of adaptation is important when considering public policies for the whole population. One factor that limited adherence to PA was the self-perception of fair or poor health. Given the cross-sectional nature of our study, a causal relationship between these two variables could not be established. However, the perception of poor health may represent a robust negative psychological influence on exercise engagement. The fear of worsening previous diseases may be stronger than the appeal of attaining the well-known benefits of exercise on health status. Conversely, we observed that individuals who self-reported hypertension, diabetes, and dyslipidemia were more likely to engage in LTPA when they had better self-perceived health status. Alkerwi et al. ${ }^{29}$ have shown that, in three European regions, the awareness of the positive health effects of PA may be crucial to motivating persons to become more active. Therefore, the benefits of regular exercise for chronic diseases must be better explained to those with and without a positive self-perception of health.

The BMI also affected adherence to PA in all three groups investigated. We found an inverse relationship between PA and BMI in men and women. Again, the subgroup that would receive the greatest benefit from exercise (i.e., those with higher BMI) showed lower adherence to PA. Healthy adults have been reported to perform more PA compared to unhealthy obese adults ${ }^{30}$. The odds of inactivity were higher for women with a higher BMI, lower for former smokers, and higher for current smokers ${ }^{28}$. Our study also indicated that regular smoking could be associated with low adherence to PA recommendations. The results of Churilla et al. ${ }^{7}$ have also shown that the prevalence of inactivity was greater among current smokers than in former or never smokers.

Participants with a negative self-perception of their conditions and those who did not have the opportunity to exercise in their neighborhood had lower adherence to PA. Therefore, our data support the importance of improving urban mobility and the provision of adequate facilities to help persons practice PA close to where they live, especially for priority groups, such as older adults, individuals who are obese, and individuals who have diabetes, hypertension, and other chronic diseases.

In summary, the proportion of adults with diabetes, dyslipidemia, and hypertension who were adherent to the PA recommendations remained low. Only one out of five received the benefits that PA could have for their health. Men were more active than women when these diseases were present. The main conditions that favored adherence to PA were higher levels of income and education. Subjects with a negative self-perception of their health and those who did not have the opportunity to exercise in their neighborhood also showed lower adherence to PA. Older age, excessive body weight, fair self-perceived health, and regular smoking were also associated with decreased adherence. These findings are crucial for helping decision makers implement effective prevention strategies, target high-risk groups, and control and treat chronic diseases.

\section{Study Limitations}

Our study has some limitations. Physical activity was evaluated by a questionnaire rather than by objective measurements. Moreover, PA in this study included only exercise performed during leisure time. Therefore, it did not include all energy expenditures, which could be from active transportation (e.g., walking, biking), household work, or occupational work. Moreover, the cross-sectional nature of the data allowed associations but not causal inferences. Finally, we used self-report to determine dyslipidemia, hypertension, and diabetes because the main objective of the study was to evaluate adherence to PA in individuals aware of these chronic conditions. However, this strategy may have excluded a substantial number of undiagnosed subjects. 


\section{REFERENCES}

1. Mendis S, Davis S, Norrving B. Organizational update: the World Health Grganization Global Status Report on Noncommunicable Diseases 2014; one more landmark step in the combat against stroke and vascular disease. Stroke. 2015;46(5):e121-2. https://doi.org/10.1161/STROKEAHA.115.008097

2. Feigin VL, Roth GA, Naghavi M, Parmar P, Krishnamurthi R, Chugh S, et al. Global burden of stroke and risk factors in 188 countries, during 1990-2013: a systematic analysis for the Global Burden of Disease Study 2013. Lancet Neurol. 2016;15(9):913-24. https://doi.org/10.1016/S1474-4422(16)30073-4

3. Thompson D, Walhin JP, Batterham AM, Stokes KA, Cooper AR, Andrews RC. Effect of diet or diet plus physical activity versus usual care on inflammatory markers in patients with newly diagnosed type 2 diabetes: the Early ACTivity in Diabetes (ACTID) randomized, controlled trial. J Am Heart Assoc. 2014;3(3):e000828. https://doi.org/10.1161/JAHA.114.000828

4. World Health Organization. Global recommendations on physical activity for health. Geneva: WHO; 2010 [cited 2017 Nov 28]. Available from: http://apps.who.int/iris/ bitstream/10665/44399/1/9789241599979_eng.pdf

5. Chen Y, Sloan FA, Yashkin AP. Adherence to diabetes guidelines for screening, physical activity and medication and onset of complications and death. J Diabetes Complications. 2015;29(8):1228-33. https://doi.org/10.1016/j.jdiacomp.2015.07.005

6. Umpierre D, Ribeiro PA, Kramer CK, Leitão CB, Zucatti AT, Azevedo MJ, et al. Physical activity advice only or structured exercise training and association with $\mathrm{HbA1c}$ levels in type 2 diabetes: a systematic review and meta-analysis. JAMA. 2011;305(17):1790-9. https://doi.org/10.1001/jama.2011.576

7. Churilla JR, Johnson TM, Zippel EA. Association of physical activity volume and hypercholesterolemia in US adults. QJM. 2013;106(4):333-40. https://doi.org/10.1093/qjmed/hcs231

8. Hallal PC, Andersen LB, Bull FC, Guthold R, Haskell W, Ekelund U, et al. Global physical activity levels: surveillance progress, pitfalls, and prospects. Lancet. 2012;380(9838):247-57. https://doi.org/10.1016/S0140-6736(12)60646-1

9. Knuth AG, Bacchieri G, Victora CG, Hallal PC. Changes in physical activity among Brazilian adults over a 5-year period. J Epidemiol Community Health. 2010;64(7):591-5. https://doi.org/10.1136/jech.2009.088526

10. McArthur D, Dumas A, Woodend K, Beach S, Stacey D. Factors influencing adherence to regular exercise in middle-aged women: a qualitative study to inform clinical practice. BMC Womens Health. 2014;14:49. https://doi.or/10.1186/1472-6874-14-49

11. Aquino EM, Barreto SM, Bensenor IM, Carvalho MS, Chor D, Duncan BB, et al. Brazilian Longitudinal Study of Adult Health (ELSA-Brasil): objectives and design. Am J Epidemiol. 2012;175(4):315-24. https://doi.org/10.1093/aje/kwr294

12. Schmidt MI, Duncan BB, Mill JG, Lotufo PA, Chor D, Barreto SM, et al. Cohort Profile: Longitudinal Study of Adult Health (ELSA-Brasil). Int J Epidemiol. 2015;44(1):68-75. https://doi.org/10.1093/ije/dyu027

13. Hallal PC, Gomez LF, Parra DC, Lobelo F, Mosquera J, Florindo AA, et al. Lessons learned after 10 years of IPAQ use in Brazil and Colombia. J Phys Act Health. 2010;7 Suppl 2:S259-64. https://doi.org/10.1123/jpah.7.s2.s259

14. Santos SM, Griep RH, Cardoso LO, Alves MGM, Fonseca MJM, Giatti L, et al. Cross-cultural adaptation and reliability of measurements on self-reported neighborhood characteristics in ELSA-Brasil]. Rev Saude Publica. 2013;47 Supl 2:122-30. https://doi.org/10.1590/S0034-8910.2013047003871

15. Franco MR, Tong A, Howard K, Sherrington C, Ferreira PH, Pinto RZ, et al. Older people's perspectives on participation in physical activity: a systematic review and thematic synthesis of qualitative literature. Br J Sports Med. 2015;49(19):1268-76. https://doi.org/10.1136/bjsports-2014-094015

16. Fang J, Keenan NL, Dai S. Fruit/vegetable intake and physical activity among adults with high cholesterol. Am J Health Behav. 2011;35(6):689-98. https://doi.org/10.5993/AJHB.35.6.5

17. Hays LM, Clark DO. Correlates of physical activity in a sample of older adults with type 2 diabetes. Diabetes Care. 1999;22(5):706-12. https://doi.org/10.2337/diacare.22.5.706 
18. World Health Organization. Global status report on noncommunicable diseases 2014. Geneva: WHO; 2014 [cited 2017 Nov 28]. Available from: http://www.who.int/nmh/publications/ncdstatus-report-2014/en/

19. Blomster JI, Chow CK, Zoungas S, Woodward M, Patel A, Poulter NR, et al. The influence of physical activity on vascular complications and mortality in patients with type 2 diabetes mellitus. Diabetes Obes Metab. 2013;15(11):1008-12. https://doi.org/10.1111/dom.12122

20. Loprinzi PD, Sng E, Addoh O. Physical activity and residual-specific mortality among adults in the United States. Med Sci Sports Exerc. 2016;48(9):1730-6. https://doi.org/10.1249/MSS.0000000000000952

21. Madden KM, Lockhart C, Cuff D, Potter TF, Meneilly GS. Short-term aerobic exercise reduces arterial stiffness in older adults with type 2 diabetes, hypertension, and hypercholesterolemia. Diabetes Care. 2009;32(8):1531-5. https://doi.org/10.2337/dc09-0149

22. Fan AZ, Ham SA, Muppidi SR, Mokdad AH. Validation of reported physical activity for cholesterol control using two different physical activity instruments. Vasc Health Risk Manag. 2009;5:649-61. https://doi.org/10.2147/VHRM.S6164

23. Pitanga CPS, Pitanga FJG, Beck CC, Gabriel RECD, Moreira MHR. [Level of physical activity in the prevention of excess visceral fat in postmenopausal women: how much is needed?]. Arq Bras Endocrinol Metabol. 2012;56(6):358-63. Portuguese. https://doi.org/10.1590/S0004-27302012000600003

24. Kanagasabai T, Thakkar NA, Kuk JL, Churilla JR, Ardern CI. Differences in physical activity domains, guideline adherence, and weight history between metabolically healthy and metabolically abnormal obese adults: a cross-sectional study. Int J Behav Nutr Phys Act. 2015;12:64. https://doi.org/10.1186/s12966-015-0227-z

25. Ribeiro AL, Duncan BB, Brant LC, Lotufo PA, Mill JG, Barreto SM. Cardiovascular health in Brazil: trends and perspectives. Circulation. 2016;133(4):422-33. https://doi.org/10.1161/CIRCULATIONAHA.114.008727

26. Jefferis BJ, Sartini C, Lee IM, Choi M, Amuzu A, Gutierrez C, et al. Adherence to physical activity guidelines in older adults, using objectively measured physical activity in a population-based study. BMC Public Health. 2014;14:382. https://doi.org/10.1186/1471-2458-14-382

27. Pitanga FJG, Lessa I, Barbosa PJB, Barbosa SJO, Costa MC, Lopes AS. Factors associated with leisure time physical inactivity in black individuals: hierarchical model. PeerJ. 2014;2:e577. https://doi.org/10.7717/peerj.577

28. Dontje ML, Krijnen WP, Greef MH, Peeters GG, Stolk RP, Schans CP, et al. Effect of diagnosis with a chronic disease on physical activity behavior in middle-aged women. Prev Med. 2016;83:56-62. https://doi.org/10.1016/j.ypmed.2015.11.030

29. Alkerwi A, Schuh B, Sauvageot N, Zannad F, Olivier A, Guillaume M, et al. Adherence to physical activity recommendations and its associated factors: an interregional population-based study. J Public Health Res. 2015;4(1):406. https://doi.org/10.4081/jphr.2015.406

30. Bell JA, Hamer M, Hees VT, Singh-Manoux A, Kivimäki M, Sabia S. Healthy obesity and objective physical activity. Am J Clin Nutr. 2015;102(2):268-75. https://doi.org/10.3945/ajcn.115.110924

Funding: The ELSA-Brasil baseline study was supported by the Brazilian Ministry of Health (Science and Technology Department) and the Brazilian Ministry of Science and Technology (CNPq - Grants 01060010.00 RS, 01060212.00 BA, 0106 0300.00 ES, 0106 0278.00 MG, 01060115.00 SP, 01060071.00 RJ).

Authors' Contribution: Design and planning of the study: MJG, GRH, SI, PF, MMCB. Analysis and interpretation of the data: FL, MJG, GRH, SI, PF, MMCB. Preparation or review of the study: FL, MJG, GRH, SI, PF, MMCB. Approval of the final version: FL, MJG, MMCB.

Conflict of Interest: The authors declare no conflict of interest. 